\title{
Kinematic and EMG Comparison Between Variations of Unilateral Squats Under Different Stabilities
}

(요 $\circledast \ominus$

\author{
Authors \\ Roland van den Tillaar, Stian Larsen
}

\begin{abstract}
Affiliations
Department of Sports Sciences and Physcial Education, Nord University, Levanger, Norway
\end{abstract}

Key words

strength, electromyography, stability, force

$\begin{array}{ll}\text { received } & 07.04 .2020 \\ \text { revised } & 02.06 .2020 \\ \text { accepted } & 05.06 .2020\end{array}$

Bibliography

DOI https://doi.org/10.1055/a-1195-1039

Sports Medicine International Open 2020; 4: E59-E66

(c) Georg Thieme Verlag KG Stuttgart · New York

ISSN 2367-1890

Correspondence

Prof. Roland van den Tillaar

Sports Sciences and Physical Education, Nord University, Levanger

Odins veg 23

7603 Levanger

Norway

Tel : 004797662913, Fax : 004774112001

roland.v.tillaar@nord.no

\section{ABSTRACT}

The purpose of the study was to compare kinematics and muscle activity between two variations of unilateral squats under different stability conditions. Twelve male volunteers (age: $23 \pm 5$ years, mass: $80 \pm 17 \mathrm{~kg}$, height: $1.81 \pm 0.11 \mathrm{~m}$, strengthtraining experience: $4.3 \pm 1.9$ years) performed four repetitions with the same external load ( $\approx 4 \mathrm{RM}$ ). Two variations (with the non-stance leg forwards vs. backwards) were performed in a Smith-machine and free-weight condition. The variables were barbell velocity, lifting time and surface electromyography activity of the lower extremity and trunk muscles during the descending and ascending phase. The main findings were 1) peak force was higher when performing the unilateral squats in the Smith machine; 2) peak ascending barbell velocity increased from repetition 3-4 with free weight; and 3) muscle activity from the rectus femoris, vastus lateral, biceps femoris, gluteus medius, and erector spinae increased with repetitions, whereas gluteus, and medial vastus and shank muscles were affected by the conditions. It was concluded that more peak force could be produced because of increased stability. However, peak barbell velocity increased from repetition to repetition in free-weight unilateral squats, which was probably because the participants grew more comfortable. Furthermore, increased instability causes more gluteus and vastus medial activation and foot variations mainly affected the calf muscles.

\section{Introduction}

The use of unilateral exercises such as step-ups, lunges, and unilateral squats are popular strength exercises to enhance maximal strength [1-3]. In general, people are capable of producing less than twice as much force bilaterally as unilaterally; this phenomenon is known as the bilateral deficit [4-6]. When the goal is to produce maximal force, the bilateral deficit is approximately $13 \%$ for the lower body. However, the evidence is not conclusive if unilateral exercises may be favourable when compared with bilateral exercises $[5,7,8]$.

Therefore, Eliassen, Saeterbakken, and van den Tillaar [2] compared the bilateral squat with two unilateral squat variations (elevated non-stance foot forwards vs. backwards) during a four-repetition maximum (4RM) on barbell kinematics and muscle activation. Findings were that muscle activity was higher for all three quadriceps muscles, the erector spinae, and biceps femoris in the ascent phase of the bilateral squat, whereas the semitendinosus had higher muscle activity in the descent phase of the unilateral squats. In addition, in a comparison of the same external load per leg, it produced greater peak vertical ground reaction force and barbell velocity in unilateral squats when compared to the bilateral squat, which is important for strength development. When the authors compared unilateral squats with the non-stance foot forwards vs. non-stance foot backwards in relation to the trunk, the foot back variation produced greater peak vertical ground reaction force.

Another study conducted by Khuu, Foch, and Lewis [9] compared how changing the position of the elevated non-stance foot affected the kinematics and kinetics in three unilateral squat variations. They found greater ipsilateral trunk flexion, pelvic anterior tilt, hip flexion and adduction, and external rotation as well as less knee flexion and abduction when the non-stance leg pointed back- 
wards compared to when the non-stance leg was neutral and pointed forwards. In addition, squatting unilaterally with the non-stance foot backwards led to greater hip external rotator moment, kneeextensor moment, less hip-extensor moment and knee adductor moment at peak knee-flexion relative to non-stance foot neutral and forward.

However, when the goal is to increase strength on the lower extremity with exercises, like the bilateral and unilateral squat, sports scientists, therapists, and coaches have often used the Smith machine during the two past decades. Arandjelovic [3] reported three reasons why the Smith machine could be beneficial in strength training rather than a free-weight barbell. Firstly, exercising on a Smith machine requires fewer motor skills. Secondly, decreasing the demands for balance with a Smith machine could, therefore, allow practitioners to train more safely with heavy loads and thirdly, constrained degrees of freedom could offer a safer environment for plyometric strength exercises. This conclusion is contradictory to when unilateral squats are performed with free weights because mastering both balance and strength becomes more important owing to the smaller support-surface for the foot.

To the authors ' knowledge, no studies have compared heavy weight ( $>80 \%$ of one-repetition maximum) unilateral squats (with non-stance leg forwards and backwards) between free-weight and Smith-machine squats when the same external load is used per leg. The non-weight-bearing foot in unilateral squats, either forwards or backwards, could influence kinematics and muscle activation due to different weight distributions [2]. Also, using a Smith machine when performing unilateral squats under the different conditions could limit the balance requirement because the vertical bar path is closed and therefore influences kinematics and muscle activation.

Therefore, the present study aimed to compare kinematics and muscle activity in unilateral squats with the non-stance leg forwards and backwards between free weights and the Smith machine at the same external load per leg. It was hypothesized that peak force output and maximal ascending barbell velocity would be greater with unilateral squats in a Smith machine compared with the free-weight squats because of the closed vertical bar path, which results in more effective muscle use to enhance force production [3]. Understanding the eventual differences in kinematics and muscle activity between the variations of unilateral squats in stable and less stable conditions could be of interest for trainers, athletes, and coaches in their training in order to understand what is emphasized when performing these variations with different stability requirements.

\section{Materials and Methods}

\section{Participants}

Twelve male participants (age: $23.1 \pm 4.5$ years, mass: $80.5 \pm 17.1 \mathrm{~kg}$, height: $1.81 \pm 0.11 \mathrm{~m}$ ) with previous resistance training experience $(4.3 \pm 1.9$ years $)$ volunteered for this study. Participants without any history of neurological or orthopaedic dysfunction, surgery or pain in the spine and lower extremities, and who had experience with unilateral squats and could lift at least 1.5 times their own body mass in bilateral squats were recruited. The participants did not perform any resistance training exercises targeting the lower extremities in the 48 hours before the testing session. The participants provided written informed consent to participate in the study, which was approved by the Norwegian Center for Research Data and performed according to the Declaration of Helsinki and of the journal [10].

\section{Experimental design}

To compare the effect of non-stance foot variations and stability conditions upon kinematics and muscle activity in unilateral squats, a within-subjects design with repeated measures was used in which each participant performed both unilateral squat variations (foot forwards and backwards) in a free-weight and Smith-machine condition. Four repetitions with a heavy load $(\approx 4 \mathrm{RM})$ in unilateral squats were used because it was a typical training load used to increase maximal strength $[11,12]$. The dependent variables were peak vertical force, barbell velocity, lifting time, and surface electromyography (EMG) activity of the lower extremity and trunk during the descending and ascending phase of all four repetitions in each condition.

\section{Procedures}

Before the test session, participants were given a two-week familiarization period (two to three training sessions) to establish four repetitions in free-weight unilateral squats with a stable technique (controlled by observation of the test leader who had years of power lifting training at the national level) and train with these loads to avoid a learning effect during testing. The load of the four repetitions during the free-weight condition was based upon 4RM load in free-weight bilateral squats with $90^{\circ}$ knee flexion (135.9 \pm $21.5 \mathrm{~kg}$ ) established in the first familiarization session. The external load was calculated by the formula ((body weight + external 4RM load) (2) - body weight. This load was based upon the study of Eliassen, Saeterbakken, and van den Tillaar [2] for safety reasons and to ensure that the participants could lift the load four times in the exercise variations and stability conditions. Between each attempt and between each squat exercise, participants were given a five-minute rest between each attempt for optimal performance [13]. The order of the two unilateral squat variations and stability conditions was randomized and counterbalanced to avoid fatigue. The participant stood at the same place in each condition on a force plate (Ergotest Technology AS, Porsgrunn, Norway) sampling at $1000 \mathrm{~Hz}$, always with the knee of the preferred foot fully extended and the opposite knee bent approximately $90^{\circ}$ (foot backwards) or fully extended but slightly elevated (foot forwards) with a barbell placed on the upper part of the shoulders. From this position, the participant flexed the knee in a controlled manner and squatted down to a $90^{\circ}$ knee angle during all lifts to reduce technique and balance requirements and to make possible a comparison of muscle activations. A $90^{\circ}$ knee angle was found using a protractor and a horizontal rubber band used to identify this lower position during the tests $[14,15]$, which the participants had to touch with the proximal part of their hamstring before starting the ascending movement ( $>$ Fig. 1). The participants were instructed to descend at their preferred tempo (to avoid extra stress and increase the external validity towards training), but the ascending movement had to be performed at maximal velocity during every repetition in each of the squat conditions. The heel was in contact with the floor at all 

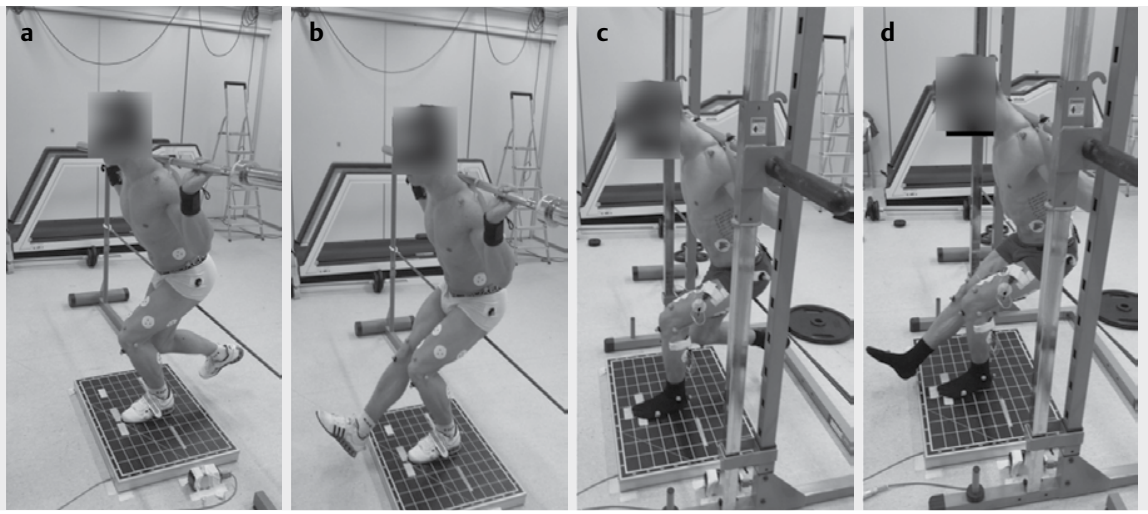

-Fig. 1 Unilateral free-weight squats with foot backwards (a), forwards (b), and in Smith machine (c and d).

times. The 4RM load in the free-weight lifts was also used as a load in the Smith machine to investigate the effect of the same external load when making the condition more stable owing to the closed vertical bar path, and therefore decreasing the balance requirement.

Prior to data collection, participants performed a five-minute jog as a general warm up followed by a specific warm-up consisting of 1) 10 repetitions of bilateral squats without extra load, 2) 10 repetitions with the barbell $(20 \mathrm{~kg}), 3) 10$ repetitions with $50 \%$ of $1 \mathrm{RM}$, and 4) 6 repetitions with $70 \%$ of $1 \mathrm{RM}$ [2].

A linear encoder sampling at $500 \mathrm{~Hz}$ (ET-Enc-02, Ergotest Technology AS, Porsgrunn, Norway) connected to the barbell measured the vertical position and velocity (barbell kinematics) together with the peak force measured by the force plate. The velocity of the barbell was calculated by using a 5-point differential filter with the Musclelab V10.6 software (Ergotest Technology AS).

Based on the recommendations of SENIAM [16], Musclelab v.10.5.60 (Ergotest Technology AS) was used to measure electromyographic (EMG) activity in eleven muscles: a) vastus medialis, b) vastus lateralis, c) rectus femoris, d) lateral side of the gastrocnemius, e) gluteus maximus, f) gluteus medius, g) external abdominal oblique, h) erector spinae at $L 4-L 5$, i) semitendinosus, j) the long head of the biceps femoris, and k) soleus. The skin was prepared by shaving, abrading, and cleaning with isopropyl alcohol to reduce skin impendence before positioning the electrodes over each muscle. To strengthen the signal, the conductive gel was applied to self-adhesive electrodes (Dri-Stick Silver circular sEMG Electrodes AE-131; NeuroDyne Medical, Cambridge, MA, USA). The electrodes (11-mm contact diameter, 20-mm centre-to-centre distance) were placed on the participant's stance side used in the unilateral squats. To minimize noise induced from external sources, the EMG raw signal was amplified and filtered using a preamplifier located as near to the pickup point as possible. The common-mode rejection ratio (CMRR) was $106 \mathrm{~dB}$, and the input impedance between each electrode pair was $>10^{12} \Omega$. The EMG signals were sampled at a rate of $1000 \mathrm{~Hz}$. Signals were band-pass filtered (fourthorder Butterworth filter) with a cut-off frequency of $20 \mathrm{~Hz}$ and 500 $\mathrm{Hz}$, rectified, integrated, and converted to root-mean-square (RMS) signals using a hardware circuit network (frequency response 450 $\mathrm{kHz}$, averaging constant $12 \mathrm{~ms}$, total error $\pm 0.5 \%$ ) [12]. To locate possible differences in muscle activity during the squat exercises, the average RMS was calculated for the ascending phase for each repetition because the maximal muscle activation was detected in this part [17]. Only the last three repetitions were used for further analysis because the first repetition differs significantly from the others based upon earlier studies [18]. The phases were identified with the linear encoder and force plate, which were synchronized with the EMG recordings using Musclelab V10.5 (Ergotest Technology AS).

\section{Statistical analyses}

To assess the differences in barbell kinematics between the two squat variations and conditions, a repeated 2 (condition: free weight, Smith machine) $\times 2$ (variation: squat with foot forwards vs. foot backwards) $\times 3$ (repetition: 2-4) analysis of variance (threeway ANOVA) design on the descending and ascending phase was used. For muscle activity and peak force, a 2 (condition: free weight, Smith machine) $\times 2$ (variation: squat with foot forward vs. foot backwards) $\times 3$ (repetition: 2-4) ANOVA on the ascending phase was performed. Holm-Bonferroni post hoc tests were used to identify the differences in barbell kinematics and EMG activity of the 11 muscles. If the sphericity assumption was violated, the Greenhouse-Geisser adjustments of the p-values were reported. All results are presented as mean \pm SEM. The level of significance was set at $p<0.05$. The effect size was evaluated with $\eta^{2}$ (eta partial squared), where $0.01<\eta^{2}<0.06$ constitutes a small effect, $0.06<\eta^{2}<0.14$ a medium effect, and $\eta^{2}>0.14$ a large effect [19]. Statistical analyses were performed in SPSS version 26.0 (IBM Corp., Armonk, NY, USA).

\section{Results}

The total external load during the unilateral squats was $27.9 \pm 11.4$ $\mathrm{kg}$. A significant effect of condition $\left(F=7.1, p=0.022, \eta^{2}=0.39\right)$ was found for peak force together with a significant condition * repetition effect $\left(F=5.9, p=0.009, \eta^{2}=0.35\right)$. Post hoc comparison revealed that peak force was higher when performing the unilateral squats in the Smith-machine with the foot forward compared with the same variation with free weights. Furthermore, the peak force between repetitions in the Smith machine was stable, whereas it slightly increased in the free-weight squat condition from repetition 3-4 (• Fig. 2). 
The lifting time was not significantly affected by foot position or lifting condition in the descending and ascending phases $\left(F \leq 3.52, p \geq 0.08, \eta^{2} \leq 0.24\right)$. Both descending and ascending barbell peak velocities were significantly affected by repetition and condition * repetition interaction effect $\left(F \geq 4.3, p \leq 0.026, \eta^{2} \geq 0.28\right)$. In addition, peak ascending barbell velocity was affected by the condition $\left(F=8.0, p=0.017, \eta^{2}=0.42\right)$. Post hoc comparison revealed that the maximal ascending barbell velocity increased from repetition 3-4, but only when performing with the foot forwards in both conditions. Furthermore, a significant difference was noted between the Smith-machine foot-backwards condition and the two free-weight variations in repetition 4. Maximal descending barbell velocity increased over repetitions in free-weight conditions but did not change in the Smith-machine condition (- Fig. 3).
A significant effect of repetition ( $\left.F \geq 4.3, p \leq 0.026, \eta^{2} \geq 0.28\right)$ was found for the rectus femoris, lateral vastus, biceps femoris, gluteus medius, and erector spinae ( $\vee$ Tables $\mathbf{1}$ and $\triangleright \mathbf{2}$ ). In addition, a significant effect of condition $\left(F \geq 3.2, p \leq 0.039, \eta^{2} \geq 0.24\right)$ was found for the vastus medialis, gluteus medius and maximus, gastrocnemius, and soleus. No significant interaction effects were found ( $\left.F \leq 1.1, p \geq 0.35, \eta^{2} \leq 0.10\right)$. Post hoc comparison revealed that muscle activation increased significantly from repetition 2-4 for the rectus femoris, lateral vastus, biceps femoris, and erector spinae, whereas it increased significantly from repetition 3-4 for the lateral vastus, gluteus medius, and erector spinae ( $>$ Tables 1 and $>2$ ). However, each exercise was evaluated, only a few significant increases between repetitions were found, mainly the freeweight conditions in the lower limb muscles ( $\triangleright$ Table 1 ) and the

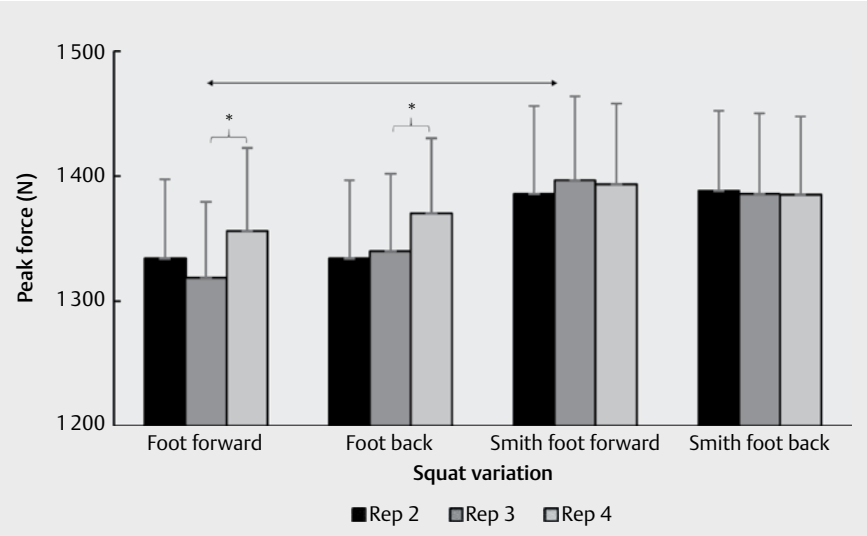

-Fig. 2 Mean (SEM) peak force during unilateral free-weight squats with foot backwards and forwards and in Smith machine. * indicates a significant difference between these two repetitions on a $p<0.05$ level. $\leftrightarrow$ Indicates a significant difference between the two conditions on a $p<0.05$ level.
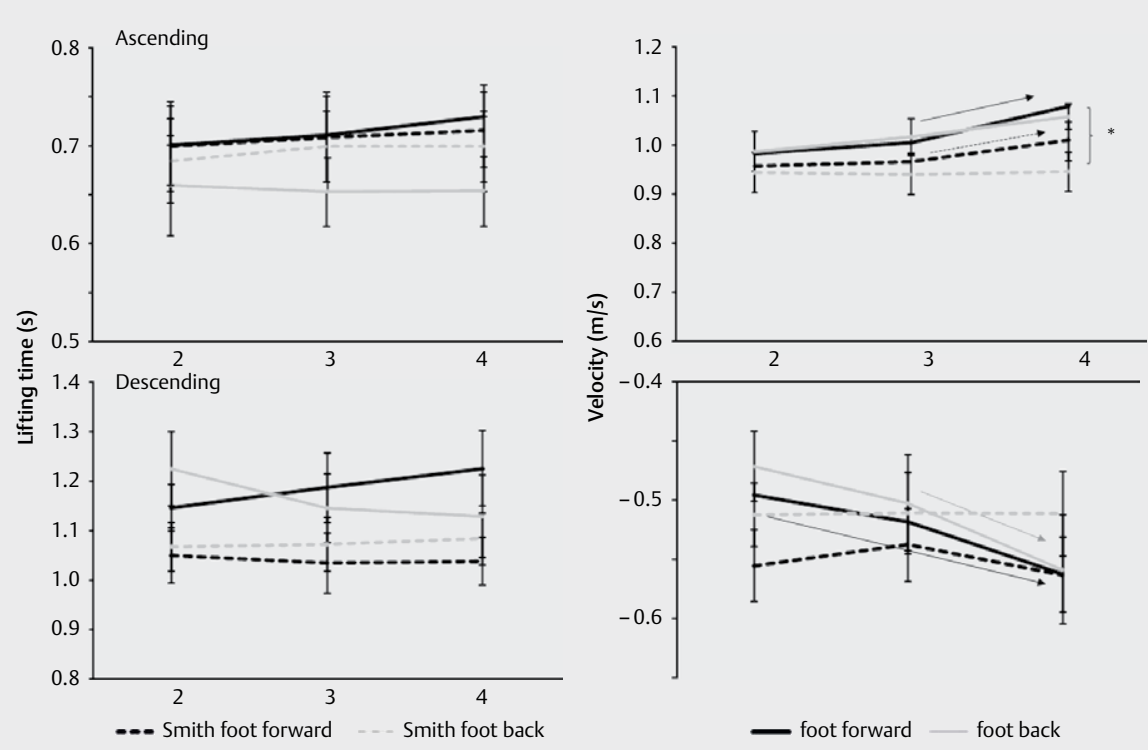

- Fig. 3 Mean (SEM) lifting time and peak velocity during the descending and ascending phase in unilateral free-weight squats with foot backwards and forwards and in Smith machine. ${ }^{*}$ indicates a significant difference between Smith machine with foot backwards and the two free-weight variations in repetition 4 on a $p<0.05$ level. $\rightarrow$ indicates a significant difference between these two repetitions for this squat variation on a $p<0.05$ level. 
- Table 1 Mean RMS ( \pm SEM) EMG activity of the seven lower limb muscles for repetitions 2-4 in the descending and ascending phase of the free-weight and Smith-machine squats with foot forward (F.F.) and back F.B.

\begin{tabular}{|c|c|c|c|c|c|c|c|}
\hline \multirow[t]{2}{*}{ Muscle $(\mu \mathrm{V})$} & \multirow[t]{2}{*}{ Condition } & \multicolumn{3}{|c|}{ Repetition } & \multirow{2}{*}{$\begin{array}{l}\text { Significance } \\
\text { between reps }\end{array}$} & \multirow{2}{*}{$\begin{array}{l}\text { Averaged over } \\
\text { repetitions }\end{array}$} & \multirow{2}{*}{$\begin{array}{l}\text { Significance between } \\
\text { conditions }\end{array}$} \\
\hline & & 2 & 3 & 4 & & & \\
\hline \multirow[t]{4}{*}{ Rectus femoris } & Free weight F.F. & $165 \pm 24$ & $176 \pm 22$ & $192 \pm 28$ & & $177 \pm 24$ & \\
\hline & Free weight F.B. & $163 \pm 24$ & $180 \pm 32$ & $193 \pm 27$ & 2 with 4 & $179 \pm 27$ & \\
\hline & Smith machine F.F. & $160 \pm 26$ & $158 \pm 26$ & $175 \pm 27$ & & $164 \pm 25$ & \\
\hline & Smith machine F.B. & $179 \pm 30$ & $183 \pm 33$ & $191 \pm 31$ & & $184 \pm 31$ & \\
\hline \multirow[t]{4}{*}{ Vastus medialis } & Free weight F.F. & $294 \pm 54$ & $300 \pm 48$ & $304 \pm 53$ & & $299 \pm 51$ & \\
\hline & Free weight F.B. & $271 \pm 56$ & $271 \pm 56$ & $285 \pm 50$ & & $276 \pm 54$ & \\
\hline & Smith machine F.F. & $269 \pm 44$ & $263 \pm 46$ & $271 \pm 43$ & & $268 \pm 44$ & \\
\hline & Smith machine F.B. & $246 \pm 46$ & $251 \pm 50$ & $255 \pm 49$ & & $251 \pm 48^{*}$ & with free-weight conditions \\
\hline \multirow[t]{4}{*}{ Vastus lateralis } & Free weight F.F. & $272 \pm 32$ & $292 \pm 34$ & $306 \pm 39$ & 2 with 4 & $290 \pm 34$ & \\
\hline & Free weight F.B. & $270 \pm 31$ & $259 \pm 29$ & $293 \pm 37$ & 3 with 4 & $274 \pm 32$ & \\
\hline & Smith machine F.F. & $265 \pm 29$ & $257 \pm 25$ & $274 \pm 28$ & & $265 \pm 26$ & \\
\hline & Smith machine F.B. & $231 \pm 31$ & $244 \pm 33$ & $252 \pm 39$ & & $242 \pm 34$ & \\
\hline \multirow[t]{4}{*}{ Semitendinosus } & Free weight F.F. & $90 \pm 16$ & $87 \pm 15$ & $92 \pm 17$ & & $90 \pm 15$ & \\
\hline & Free weight F.B. & $77 \pm 15$ & $87 \pm 18$ & $89 \pm 18$ & & $90 \pm 17$ & \\
\hline & Smith machine F.F. & $73 \pm 12$ & $75 \pm 12$ & $85 \pm 16$ & & $77 \pm 13$ & \\
\hline & Smith machine F.B. & $68 \pm 11$ & $74 \pm 13$ & $78 \pm 13$ & & $73 \pm 11$ & \\
\hline \multirow[t]{4}{*}{ Biceps femoris } & Free weight F.F. & $91 \pm 16$ & $102 \pm 21$ & $112 \pm 19$ & 2 with 4 & $102 \pm 18$ & \\
\hline & Free weight F.B. & $99 \pm 17$ & $104 \pm 17$ & $106 \pm 20$ & & $103 \pm 17$ & \\
\hline & Smith machine F.F. & $87 \pm 15$ & $94 \pm 17$ & $96 \pm 17$ & 2 with 4 & $92 \pm 16$ & \\
\hline & Smith machine F.B. & $105 \pm 20$ & $103 \pm 20$ & $114 \pm 24$ & & $107 \pm 21$ & \\
\hline \multirow[t]{4}{*}{ Soleus } & Free weight F.F. & $88 \pm 14$ & $79 \pm 12$ & $87 \pm 12$ & & $99 \pm 18$ & \\
\hline & Free weight F.B. & $91 \pm 3$ & $83 \pm 11$ & $100 \pm 11$ & & $117 \pm 30$ & \\
\hline & Smith machine F.F. & $104 \pm 11$ & $108 \pm 14$ & $111 \pm 14$ & & $122 \pm 18^{*}$ & $\begin{array}{l}\text { with free-weight foot forward } \\
\text { and Smith-machine foot back }\end{array}$ \\
\hline & Smith machine F.B. & $76 \pm 10$ & $81 \pm 10$ & $86 \pm 13$ & & $84 \pm 10$ & \\
\hline \multirow[t]{4}{*}{ Gastrocnemius } & Free weight F.F. & $69 \pm 12$ & $61 \pm 9$ & $70 \pm 10$ & & $67 \pm 9$ & \\
\hline & Free weight F.B. & $62 \pm 14$ & $63 \pm 12$ & $64 \pm 11$ & & $63 \pm 11$ & \\
\hline & Smith machine F.F. & $71 \pm 10$ & $66 \pm 8$ & $67 \pm 9$ & & $68 \pm 8$ & \\
\hline & Smith machine F.B. & $43 \pm 7$ & $45 \pm 7$ & $42 \pm 7$ & & $43 \pm 6^{*}$ & with foot forward conditions \\
\hline
\end{tabular}

Smith-machine conditions in trunk muscles ( $\vee$ Table 2 ). A post hoc comparison for condition showed that muscle activity of the gluteus maximus and vastus medialis in the Smith machine with foot backwards were significantly lower than in the free-weight conditions, whereas gastrocnemius activity was lower in the Smith machine with the foot backwards compared with all foot-forwards conditions. Gluteus medius activity in the Smith machine with foot forwards was lower than with the free-weight foot-forwards condition, whereas soleus activity was significantly higher when performing a unilateral squat with the foot forwards in the Smith machine compared with the foot-backwards condition in the Smith machine and with the free-weight foot-forwards condition ( $\bullet$ Table 1 ).

\section{Discussion}

The present study compared kinematics and muscle activity in four unilateral squat exercises with the same external load for resistance-trained participants. The main findings were that peak force was higher when performing the unilateral squats in the Smith ma- chine and that peak ascending barbell velocity increased from repetition 3-4 when performing with the foot forwards in both conditions. Furthermore, muscle activity from the rectus femoris, vastus lateral, biceps femoris, gluteus medius, and erector spinae increased during the last repetition, whereas both gluteus muscles and vastus medialis had in general lower activation in the Smithmachine condition than the free-weight conditions. The gastrocnemius had lower activation in the Smith machine with the foot backwards compared with all foot-forwards conditions, whereas the soleus had higher activation in the Smith machine with the foot forwards compared with the foot-backwards condition in the Smith machine and with the free-weight foot-forwards condition.

In the free-weight conditions, peak vertical force increased from repetition 3-4 ( $\vee$ Fig. 2). A plausible explanation for this is that both free-weight conditions had an increase in peak descent velocity from repetition 3-4 resulting in higher peak forces [20], thereby causing a higher ground reaction force. In addition, the Smith-machine foot-forwards condition had a significantly higher peak force compared to the free-weight foot-forwards condition. This differ- 
- Table 2 Mean RMS ( \pm SEM) EMG activity of the gluteus and trunk muscles for repetitions 2-4 in the descending and ascending phase of the free-weight and Smith-machine squats with foot forward (F.F.) and back F.B.

\begin{tabular}{|c|c|c|c|c|c|c|c|}
\hline \multirow[t]{2}{*}{ Muscle $(\mu \mathrm{V})$} & \multirow[t]{2}{*}{ Condition } & \multicolumn{3}{|c|}{ Repetition } & \multirow{2}{*}{$\begin{array}{l}\text { Significance } \\
\text { between reps }\end{array}$} & \multirow{2}{*}{$\begin{array}{l}\text { Averaged over } \\
\text { repetitions }\end{array}$} & \multirow{2}{*}{$\begin{array}{l}\text { Significance } \\
\text { between } \\
\text { conditions }\end{array}$} \\
\hline & & 2 & 3 & 4 & & & \\
\hline \multirow[t]{4}{*}{ Gluteus medius } & Free weight F.F. & $157 \pm 22$ & $159 \pm 23$ & $168 \pm 20$ & & $161 \pm 21$ & \\
\hline & Free weight F.B. & $159 \pm 23$ & $156 \pm 19$ & $158 \pm 18$ & & $158 \pm 20$ & \\
\hline & Smith machine F.F. & $145 \pm 20$ & $138 \pm 18$ & $149 \pm 18$ & 3 with 4 & $144 \pm 19^{*}$ & $\begin{array}{l}\text { with free-weight } \\
\text { foot forward }\end{array}$ \\
\hline & Smith machine F.B. & $150 \pm 22$ & $143 \pm 20$ & $160 \pm 24$ & 3 with 4 & $151 \pm 22$ & \\
\hline \multirow[t]{4}{*}{ Gluteus maximus } & Free weight F.F. & $153 \pm 29$ & $170 \pm 34$ & $168 \pm 32$ & & $164 \pm 31$ & \\
\hline & Free weight F.B. & $155 \pm 30$ & $164 \pm 30$ & $169 \pm 32$ & & $163 \pm 30$ & \\
\hline & Smith machine F.F. & $129 \pm 26$ & $127 \pm 25$ & $137 \pm 29$ & & $131 \pm 27$ & \\
\hline & Smith machine F.B. & $105 \pm 24$ & $111 \pm 27$ & $127 \pm 37$ & & $115 \pm 29 *$ & $\begin{array}{l}\text { with free-weight } \\
\text { conditions }\end{array}$ \\
\hline \multirow[t]{4}{*}{ Erector spinae } & Free weight F.F. & $94 \pm 19$ & $98 \pm 24$ & $113 \pm 28$ & 3 with 4 & $102 \pm 23$ & \\
\hline & Free weight F.B. & $107 \pm 24$ & $109 \pm 28$ & $121 \pm 25$ & & $112 \pm 26$ & \\
\hline & Smith machine F.F. & $67 \pm 14$ & $70 \pm 16$ & $73 \pm 16$ & & $70 \pm 15$ & \\
\hline & Smith machine F.B. & $69 \pm 17$ & $74 \pm 18$ & $88 \pm 19$ & 2 with 4 & $77 \pm 17$ & \\
\hline \multirow[t]{4}{*}{ External obliques } & Free weight F.F. & $2.1 \pm 0.5$ & $2.3 \pm 0.5$ & $2.2 \pm 0.5$ & & $2.2 \pm 0.5$ & \\
\hline & Free weight F.B. & $2.4 \pm 0.5$ & $2.2 \pm 0.5$ & $2.4 \pm 0.7$ & & $2.6 \pm 0.5$ & \\
\hline & Smith machine F.F. & $1.6 \pm 0.4$ & $1.6 \pm 0.4$ & $1.7 \pm 0.3$ & & $1.6 \pm 0.4$ & \\
\hline & Smith machine F.B. & $1.6 \pm 0.3$ & $2.0 \pm 0.5$ & $2.2 \pm 0.6$ & & $1.9 \pm 0.4$ & \\
\hline
\end{tabular}

ence is probably because the Smith machine has a fixed vertical motion, which decreases the need for balancing the bar and therefore leads to more efficient vertical force production. This speculation is supported by Behm, Anderson, and Curnew [21] who found higher force production for leg extensors and plantar flexors when the surface was stable versus unstable.

Peak ascending velocity increased in both foot-forwards variations from repetition 3-4 and caused a significant difference between the free-weight condition with the Smith-machine footbackwards variation. This finding was surprising because we expected that peak ascending velocity would be higher in the Smith-machine condition compared with the free-weight condition since because forces were higher. However, this finding could perhaps be explained because the peak descent velocity significantly increased for both free-weight variations, whereas it was stable for the Smith-machine foot-backwards condition. It is conceivable that the participants because increasingly comfortable over the repetitions using the free weights compared with the Smith machine. One reason for this could be that the participants became more secure about their lifting performance and thereby increased peak descending velocity. By increasing descending velocity, peak force was also affected in the free-weight condition. Therefore, ascending velocity also increased, which aligned with earlier studies that showed that increasing descending velocity increased ascending performances [20].

These increases coincided with rectus femoris muscle activity increases from repetition 2-4 in the free-weight foot-backwards condition. In addition, the vastus lateralis increased muscle activity during repetitions for both free-weight conditions. Meanwhile, there were no significant increases during repetitions for the knee extensor muscles in the Smith-machine conditions. These findings may also be attributed to the increased descent velocity in the freeweight conditions during repetitions. Because the quadriceps muscles are shown to be most active around the bottom position during knee flexion [22], a higher descent velocity during repetitions for the free-weight condition may have caused a higher pre-activation of the quadriceps muscles because of the stretch-shortening cycle $[23,24]$.

The vastus medialis had lower muscle activity in the Smith-machine foot-backwards condition when compared to the free-weight variations. This finding coincides with Schwanbeck, Chilibeck, and Binsted [25], who also found greater EMG activity for vastus medialis free-weight squats when compared to the Smith-machine squat. However, doing squats in a Smith machine provides a more stable surface owing to a closed vertical bar path. Therefore, the findings of the present study are in contrast with previous research on quadriceps activity on stable vs. unstable surfaces, which found less EMG activity in quadriceps muscles on unstable surfaces [21]. Nevertheless, even with the same external load per leg, the freeweight unilateral squats were probably performed at a higher percentage of 4RM owing to a more unstable bar path than the Smithmachine conditions, as indicated by a lower peak force for the footforwards free-weight condition. As a result, using a lower percentage of $4 R M$ would result in lower activation of the quadriceps muscles for the Smith-machine conditions and therefore explain differences in muscle activation between the free-weight and Smith-machine conditions. Another explanation for why the muscle activity in the vastus medialis was less active in the Smith-machine foot-backwards condition could be a shift in the centre of mass. When placing the foot forwards, the centre of mass would also shift forward; participants probably had to reposition the lifted weight through less external hip rotation and more knee abduction in the most pro- 
found knee angle in the foot-forwards condition [2]. This possibility is supported by other researchers [9], who found greater internal knee adductor moment with the foot forwards than the unilateral squats with the foot backwards. To control the knee adductor moment and align the patella, the vastus medialis in the unilateral squat with foot forwards had to be more active. Foot-forwards conditions could therefore target the vastus medialis, which could be important for avoiding injuries in the $\mathrm{ACL}$ joint $[2,26]$.

The gluteus maximus muscle activity in the Smith machine with foot backwards was lower than in the free-weight conditions, whereas gluteus medius activity was lower for the Smith-machine foot-forwards condition when compared with the free-weight footforwards condition. The increase in gluteus maximus activity for the free-weight conditions was likely a result of the requirement to maintain both tibiofemoral articulation and pelvic stability. This argument is supported by Miller, Barnes, Sofo, et al. [27], who found that unilateral squat conditions with an unstable nonbearing foot cause higher muscle activation in the gluteus maximus than with a stable nonbearing foot condition. During free-weight unilateral lifts, the gluteus medius works as an agonist when performing a hip abduction. Because these squats are more unstable than the unilateral Smith-machine squats, gluteus medius muscle activity had to increase to hold the hip joint stable [28].

Both shank muscles were also affected by the foot position. When the non-involved foot is placed backwards, the torso has a less upright position compared to when the foot is placed forwards. Consequently, the knee must flex to a greater extent to hit the depth requirements [9]. Because the gastrocnemius is a biarticular muscle and functions as both a knee flexor and dorsiflexor, greater knee flexion will inhibit gastrocnemius muscle activity due to fascicle shortening [29]. The soleus also had higher muscle activity in the Smith-machine foot-forwards condition than the Smithmachine foot-backwards condition, because the soleus muscle limits the amount of ankle dorsiflexion during descent [30] and follows the pattern of the gastrocnemius synergistically in fulldepth bilateral squats. Also, there was a difference in soleus muscle activity between Smith-machine foot-forwards and the free-weight foot-forwards conditions. This difference could perhaps be explained by a closed vertical bar path for the Smith-machine condition, which could influence ankle pronation and supination. More unstable conditions could make the soleus unable to exert similar forces as under stable conditions, and therefore use a greater variety of lower limb muscles to lock the lower limbs and body into place. This speculation is supported by Wahl and Behm [31], who found $51.2 \%$ greater soleus activity when wall sits were performed to technical failure on the stable surface compared to an unstable surface.

There are some limitations to this study. Firstly, the participants were resistance-trained but not very familiar with the different variations. Therefore, the lack of adequate experience could influence the results of the present study [32]. Even if the participants were resistance-trained, the result may not be generalized to populations who are well-trained in these specific exercises. Secondly, no kinematic analysis of the lower or trunk extremities was performed. A kinematic analysis would give more relevant information about the joint angles and velocities. It could, therefore, give more insight into why some muscles were affected by the squat condition and others not. Future studies should include kinematic analysis of these squat variations. In addition, a kinetic analysis of peak joint moments between the variations in both the ascent and descent phase could provide more practical information about the effect of these variations upon the different joints for injury prevention to coaches, athletes, and clinicians.

\section{Conclusion}

Based on the findings of the present study, it was concluded that more peak force could be produced when performing unilateral squats in a Smith machine compared with free-weight lifts due to the increased stability. However, peak barbell velocity increased from repetition to repetition in free-weight unilateral squats, which probably resulted from an increased level of comfort of the participants in this situation. Furthermore, it was concluded that freeweight unilateral squats mainly cause more gluteus maximus and medius and vastus medial activation during lifts because of increased instability, whereas foot variations mainly affect the calf muscles. Depending upon the training targets of the athletes and coaches, the authors suggest using all four variations of unilateral squats because all have power output (force * velocity) and are slightly different in muscle use.

\section{Conflict of Interest}

The authors declare that they have no conflict of interest.

\section{References}

[1] Speirs DE, Bennett MA, Finn CV et al. Unilateral vs. bilateral squat training for strength, sprints, and agility in academy rugby players. J Strength Cond Res 2016; 30: 386-392

[2] Eliassen W, Saeterbakken AH, van den Tillaar R. Comparison of bilateral and unilateral squat exercises on barbell kinematics and muscle activation. Int J Sports Phys Ther 2018; 13: 871

[3] Arandjelovic O. Common variants of the resistance mechanism in the Smith machine: Analysis of mechanical loading characteristics and application to strength-oriented and hypertrophy-oriented training. J Strength Cond Res 2012; 26: 350-363

[4] Nijem RM, Galpin A. Unilateral versus bilateral exercise and the role of the bilateral force deficit. Strength Cond J 2014; 36: 113-118

[5] Rejc E, Lazzer S, Antonutto G et al. Bilateral deficit and EMG activity during explosive lower limb contractions against different overloads. Eur J Appl Physiol 2010; 108: 157-165. doi: 10.1007/s00421-009-1199-y

[6] Oda S, Moritani T. Maximal isometric force and neural activity during bilateral and unilateral elbow flexion in humans. Eur J Appl Physiol Occup Physiol 1994; 69: 240-243. doi: 10.1007/bf01094795

[7] Ebben W, Feldmann C, Dayne A et al. Muscle activation during lower body resistance training. Int J Sports Med 2009; 30: 1-8

[8] Behm DG, Power KE, Drinkwater E. Muscle activation is enhanced with multi-and uni-articular bilateral versus unilateral contractions. Can J Appl Physiol 2003; 28: 38-52

[9] Khuu A, Foch E, Lewis C. Not all single leg squats are equal: $A$ biomechanical comparison of three variations. In J Sports Phys Ther 2016; 11: 201 
[10] Harriss D], MacSween A, Atkinson G. Ethical standards in sport and exercise science research: 2020 update. Int J Sports Med 2019; 40: 813-817. doi: 10.1055/a-1015-3123

[11] ACSM American College of Sports Medicine position stand. Progression models in resistance training for healthy adults. Med Sci Sports Exerc 2009; 41: 687-708. doi: 10.1249/ MSS.0b013e3181915670

[12] van den Tillaar R, Saeterbakken A. Effect of fatigue upon performance and electromyographic activity in 6-RM bench press. J Hum Kinet 2014; 40: 57-65. doi: 10.2478/hukin-2014-0007

[13] Rahimi R. Effect of different rest intervals on the exercise volume completed during squat bouts. J Sports Sci Med 2005; 4: 361-366

[14] Saeterbakken AH, Andersen V, van den Tillaar R. Comparison of kinematics and muscle activation in free-weight back squat with and without elastic bands. J Strength Cond Res 2016; 30: 945-952. doi: 10.1519/jsc.0000000000001178

[15] van den Tillaar R, Andersen V, Saeterbakken AH. The existence of a sticking region in free weight squats. J Hum Kinet 2014; 42: 63-71. doi: 10.2478/hukin-2014-0061

[16] Hermens HJ, Freriks B, Disselhorst-Klug C et al. Development of recommendations for SEMG sensors and sensor placement procedures. J Electromyogr Kinesiol 2000; 10: 361-374

[17] van den Tillaar R, Andersen V, Saeterbakken AH. Comparison of muscle activation and kinematics during free-weight back squats with different loads. PLoS One 2019; 14: e0217044. doi: 10.1371/journal. pone. 0217044

[18] van den Tillaar R, Andersen V, Saeterbakken A. Comparison of muscle activation and performance during $6 \mathrm{RM}$, two legged free-weight squats. Kinesiol Slov 2014; 20: 5-16

[19] Cohen J. Statistical Power Analysis for the Behavioral Sciences. Hillsdale, NJ; England: Lawrence Erlbaum Associates; 1988

[20] van den Tillaar R. Effect of descent velocity upon muscle activation and performance in two-legged free weight back squats. Sports. 2019; 7. doi: 10.3390/sports7010015
[21] Behm DG, Anderson K, RSJTJoS Curnew. Muscle force and activation under stable and unstable conditions. J Strength Cond Res 2002; 16: 416-422

[22] Isear JA, Erickson JC, Worrell TW. EMG analysis of lower extremity muscle recruitment patterns during an unloaded squat. Med Sci Sports Exerc 1997; 29: 532-539

[23] Bobbert MF, Casius LJR. Is the effect of a countermovement on jump height due to active state development? Med Sci Sports Exerc 2005; 37: $440-446$

[24] Bobbert MF, Gerritsen KG, Litjens MC et al. Why is countermovement jump height greater than squat jump height? Med Sci Sports Exerc 1996; 28: 1402-1412

[25] Schwanbeck S, Chilibeck PD, Binsted G. A comparison of free weight squat to Smith machine squat using electromyography. J Strength Cond Res 2009; 23: 2588-2591

[26] Zebis MK, Andersen LL, Bencke J et al. Identification of athletes at future risk of anterior cruciate ligament ruptures by neuromuscular screening. Am J Sports Med 2009; 37: 1967-1973

[27] Miller WM, Barnes JT, Sofo SS et al. Comparison of myoelectric activity during a suspension-based and traditional split squat. J Strength Cond Res 2019; 33: 3236-3241

[28] Boren K, Conrey C, Le Coguic J et al. Electromyographic analysis of gluteus medius and gluteus maximus during rehabilitation exercises. Int J Sports Phys Ther 2011; 6: 206

[29] Lauber B, Lichtwark GA, Cresswell AG. Reciprocal activation of gastrocnemius and soleus motor units is associated with fascicle length change during knee flexion. Physiol Rep 2014; 2: e12044

[30] Robertson D, Wilson J-M], TAS] Pierre. Lower extremity muscle functions during full squats. J Appl Biomech 2008; 24: 333-339

[31] Wahl M], Behm DG. Not all instability training devices enhance muscle activation in highly resistance-trained individuals. J Strength Cond Res 2008; 22: 1360-1370

[32] Sale DG. Neural adaptation to resistance training. Med Sci Sports Exerc 1988; 20: S135-S145 